\title{
Session 28 Overview:
}

\section{Biological Sensors for Point of Care}

\section{IIMIMD SUBCOMIMITTEE}

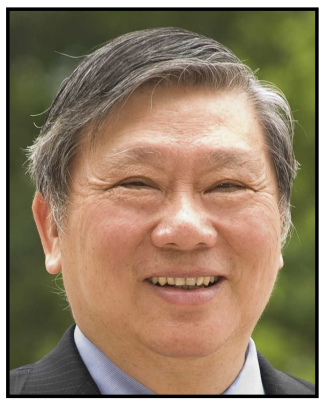

Session Chair: Peter (Chung-Yu) Wu, National Chiao Tung University, Hsinchu, Taiwan

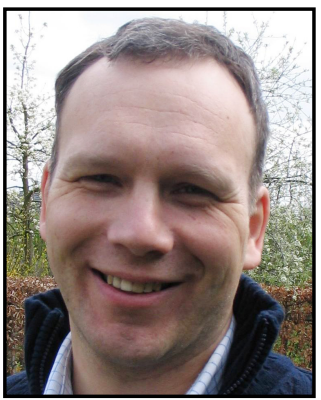

Session Co-Chair: Jan Genoe, imec, Leuven, Belgium

Point-of-care (PoC) medical services aim to deliver care at the convenient proximity of patients using dedicated PoC testing tools. Thus, they inevitably require various integrated and miniaturized biological sensors. The sensor systems discussed in this session comprise the technologies and designs for several key modalities, including: NMR (Nuclear Magnetic Resonance), ESR (Electron spin resonance), dielectric spectroscopy of water comprising biomolecules, ECG (Electrocardiogram), BIOZ (bio-impedance), PPG (Photoplethysmogram), and GSR (Galvanic Skin Response), measurements of vital signals, and airborne particle matter.

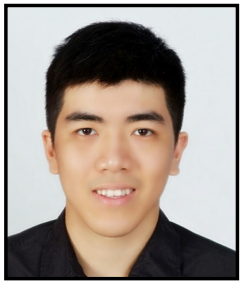

1:30 PM

28.1 A Handheld 50pM-Sensitivity Micro-NMR CMOS Platform with B-Field Stabilization for Multi-Type Biological/Chemical Assays

K-M. Lei, University of Macau, Macau, China

In Paper 28.1, the University of Macau, together with the University of Glasgow, the University of Pavia and the Instituto Superior Técnico present the first micro-NMR CMOS system for multi-type biological and chemical assays. The implementation, supported by a vertical Hall sensor and a thermal-controlled coil, reaches a detection limit of 50pM.

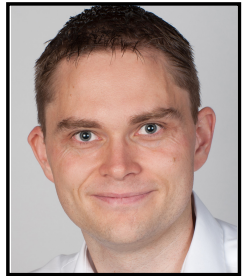

\section{2:00 PIM}

28.2 A 14GHz Battery-Operated Point-of-Care ESR Spectrometer Based on a $0.13 \mu \mathrm{m}$ CMOS ASIC

J. Anders, University of UIm, Ulm, Germany

In Paper 28.2, the University of Ulm presents a 14GHz, battery-operated, point-of-care ESR spectrometer. The portable system, containing a $0.5 \mathrm{~T}$ permanent magnet, the $15 \mathrm{~mW}$ ESR detector ASIC, and signal processing PCBs, consumes $4 \mathrm{~W}$ from a battery, which allows truly autonomous operation.

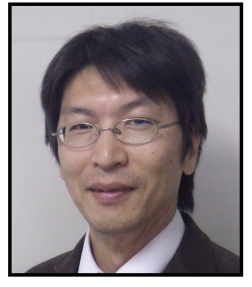

\section{2:30 PIM}

28.3 CMOS Biosensor IC Focusing on Dielectric Relaxations of Biological Water with $120 \mathrm{GHz}$ and $60 \mathrm{GHz}$ Oscillator Arrays

T. Mitsunaka, SHARP, Tenri, Japan

In Paper 28.3, Sharp, Kyoto University, and Hiroshima University propose a biosensor utilizing $60 \mathrm{GHz}$ and $120 \mathrm{GHz}$ LC oscillator arrays to detect target biological molecules in 2 dimensions by using biological water as a label. 


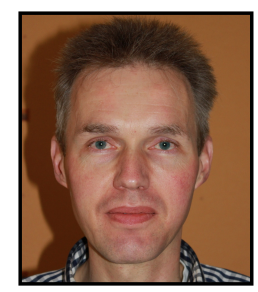

\section{3:15 PIM}

28.4 A Battery-Powered Efficient Multi-Sensor Acquisition System with Simultaneous ECG, BIO-Z, GSR, and PPG

M. Konijnenburg, Holst Centre / imec, Eindhoven, The Netherlands

In Paper 28.4, imec, the Holst Centre, and Samsung Electronics present the most complete and versatile sensor read-out system. It is the first design that supports ECG, BIOZ, PPG, and GSR. Power consumption is usually between $\sim 300$ and $1000 \mu \mathrm{W}$, depending on the application.

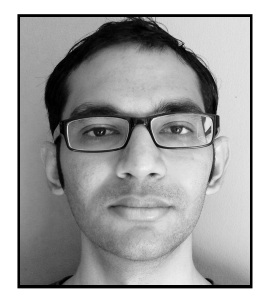

\section{$3: 45$ PIM}

28.5 A 0.6V 0.015 $\mathrm{mm}^{2}$ Time-Based Biomedical Readout for Ambulatory Applications in 40nm CMOS

R. Mohan, imec, Leuven, Belgium; KU Leuven, Leuven, Belgium

In Paper 28.5, imec, the Holst Centre, and the KU Leuven describe a time-based ECG readout front-end in $40 \mathrm{~nm}$ CMOS. It improves dynamic range by $>5 \times$ at $0.6 \mathrm{~V}$ supply while consuming only $3.3 \mu \mathrm{W}$ and occupying $0.015 \mathrm{~mm}^{2}$.

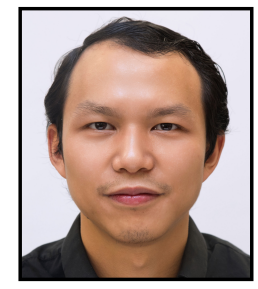

\section{4:15 PIM}

28.6 A $\pm 50 \mathrm{mV}$ Linear-Input-Range VCO-Based Neural-Recording Front-End with Digital Nonlinearity Correction

W. Jiang, University of California, Los Angeles, CA

In Paper 28.6, the University of California at Los Angeles proposes a front-end achieving a linear input-range of $\pm 50 \mathrm{mV}$, which is a $10 \times$ improvement with respect to the range of previous designs. The SFDR is $79 \mathrm{~dB}$, which is $20 \mathrm{~dB}$ higher than existing designs.

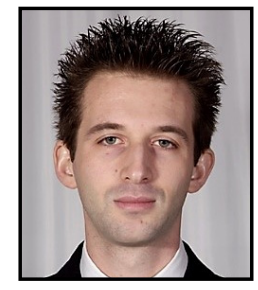

\section{4:45 PIM}

28.7 CMOS Monolithic Airborne-Particulate-Matter Detector Based on 32 Capacitive Sensors with a Resolution of $65 z \mathrm{~F}$ rms

P. Ciccarella, Politecnico di Milano, Milano, Italy

In Paper 28.7, Politecnico di Milano presents an airborne-particle-matter detector that has been integrated in CMOS technology for the first time. It implements 32 channels operating in parallel with a capacitance resolution of $65 z \mathrm{~F}, 2 \times$ better than previous single-channel CMOS capacitance detectors. 